\title{
Top-down versus bottom-up theories of phonological acquisition: A big data approach
}

\author{
Christina Bergmann ${ }^{1}$, Sho Tsuji ${ }^{1,2}$, Alejandrina Cristia ${ }^{1}$ \\ ${ }^{1}$ LSCP, Département d' Études Cognitives, ENS, EHESS, CNRS, PSL Research University, Paris, \\ France \\ ${ }^{2}$ University of Pennsylvania, Philadelphia, PA, USA \\ chbergma@gmail.com, tsujish@gmail.com, alejandrina.cristia@ens.fr
}

\begin{abstract}
Recent work has made available a number of standardized metaanalyses bearing on various aspects of infant language processing. We utilize data from two such meta-analyses (discrimination of vowel contrasts and word segmentation, i.e., recognition of word forms extracted from running speech) to assess whether the published body of empirical evidence supports a bottom-up versus a top-down theory of early phonological development by leveling the power of results from thousands of infants. We predicted that if infants can rely purely on auditory experience to develop their phonological categories, then vowel discrimination and word segmentation should develop in parallel, with the latter being potentially lagged compared to the former. However, if infants crucially rely on word form information to build their phonological categories, then development at the word level must precede the acquisition of native sound categories. Our results do not support the latter prediction. We discuss potential implications and limitations, most saliently that word forms are only one top-down level proposed to affect phonological development, with other proposals suggesting that top-down pressures emerge from lexical (i.e., wordmeaning pairs) development. This investigation also highlights general procedures by which standardized meta-analyses may be reused to answer theoretical questions spanning across phenomena.
\end{abstract}

Index Terms: language acquisition, phonology, lexical development, bottom-up, top-down, interactive, parallel

\section{Introduction}

There is a crucial theoretical quandary on top-down versus bottom-up theories of language development: Werker and Tees' [1] seminal studies demonstrated that phonological speech perception showed traces of becoming language-specific at the astoundingly young age of 12 months - which was pushed even younger for vowels by later work [2]. Since parental reports and observational studies at the time suggested that infants' lexicon at the end of the first year was vanishingly small, the last 40 years of experimental, computational, and theoretical research on infant sound category learning essentially explored bottomup theories of phonological acquisition, based mainly on the idea that there may be cues to phonological categorization readily available in infants' ambient speech experience (e.g., [3, 4]).

Top-down theories of phonological acquisition have recently experienced a revival, as several fundamental assumptions have come into question (see also [5]). To begin with, emerging results testing infants' ability to map words to objects suggest that infants comprehend a few different words already at six months $[6,7]-$ although it is an open question whether knowing 5, 10, or even 20 word-meaning mappings might suffice to affect phonological development.

An alternative is that infants may not need to know formmeaning pairs, but just the forms. A number of modeling and artificial grammar learning experiments have suggested novel ways in which infants may exploit a proto-lexicon constituted of word forms to refine phonological categories (e.g., $[8,9,10])$. For instance, Ngon et al. [11] combined infant experimentation and computational modeling to suggest that 11-month-old French learners may have up to 500 word forms in this longterm repository, and that this process relies on segmenting word forms from running speech. This work provides an empirical basis for top-down theories of phonological development, where the "top" is fed by word forms extracted from the continuous speech infants hear in their daily life.

While the above findings show possible mechanisms, they are not sufficient to prove that language acquisition takes places in a top-down way. How may we know whether infants actually profit from their word form segmentation skills to boost their phonological development? One first approach to answering this question means revisiting the actual time line of events: If infants' word segmentation skills improve before phonological categories emerge, then this lends credence to the view that there could exist a causal link in that precise direction. In contrast, if infants can develop their phonological categories by drawing directly from their auditory experience online (i.e., without an intermediate stage when the audio stream is chunked into words), then sound discrimination and word segmentation could develop in parallel. Additionally, having languagespecific phonological categories could boost infants' ability to perform word segmentation, in which case the latter could be potentially lagged compared to the former.

We note that, although diverging in terms of the relative time line of vowel and word form development, all theories agree that both of these levels should exhibit improvements as infants age. In the specific case of vowel discrimination, improvement should be evident through increases in sensitivity as a function of age for native vowels, but decreases for non-native vowels. Therefore, we can use these three predicted patterns (increases in effect size with age for word segmentation and native vowel discrimination, and decreases for non-native vowel discrimination) as a theory-independent validation test.

\subsection{Testing theories with meta-analytic data}

It is notoriously difficult to gather experimental data from infants, including measurements of their processing of spoken materials, due to the special care with which they must be recruited and tested. We have therefore proposed to re-use experimental results, and - in order to facilitate re-utilization of 
previously published (or publicly available) results - to organize them into community-augmented meta-analyses (CAMAs) [12]. In a nutshell, CAMAs are a combination of meta-analyses and public repositories centered around a specific research question. Individual entries in a CAMA correspond to experiments with groups of participants. Each entry is coded in a number of useful dimensions (including descriptors of the sample size, age, and participant native language, as well as measures of effect size). CAMAs are "community-augmented" because researchers can contribute to such databases, for instance by reporting the information necessary to calculate effect sizes that might not have been part of their manuscript, by adding new experiments and thus updating the CAMA to contain the most recent evidence, or even by reporting unpublished null-results. We have created now a number of CAMAs covering various levels of infant speech perception (e.g., [13, 14]), and have recently started combining CAMAs into standardized meta-metaanalyses using a shared platform called MetaLab $[15,16]$.

In previous work, we have investigated single phenomena when building the individual meta-analyses and CAMAs, and have thus demonstrated how such quantitative overviews of the literature can provide us with interesting answers to theoretical questions within the description of those phenomena; for instance, in [13], we measured the age at which discrimination for native versus non-native vowels differed. In the present paper, we assess whether, and how, meta-analyses may be used beyond the authors' original goals and across phenomena that concern infant speech processing with a focus on evaluating top-down versus bottom-up theories of phonological acquisition.

\section{Methods}

We drew from two meta-analyses publicly available on MetaLab, one on native and non-native vowel discrimination (henceforth Vowels-Native and Vowels-Nonnative, respectively) [13] and one covering word segmentation from native speech (WordSeg) [14]. The construction of both meta-analyses has been described elsewhere, so we limit ourselves to providing some general statistics on each dataset in Table 1, given that they have been updated after publication [12]. All scripts underlying the present analyses along with a snapshot of the data can be found at https://git.io/vHCzM.

Table 1: Description of the meta-analyses, covering both the full dataset of typically developing children and subsets limited to papers testing multiple age groups with the same materials.

\begin{tabular}{llrrr}
\hline & & \multicolumn{2}{c}{ Vowels } & WordSeg \\
& & native & non-native & \\
\hline \multirow{2}{*}{ All data: } & N Infants & 1,723 & 498 & 4,563 \\
& N Studies & 108 & 45 & 238 \\
& N Papers & 29 & 13 & 58 \\
Restricted: & N Infants & 1,051 & 379 & 1,355 \\
& N Studies & 72 & 30 & 76 \\
& N Papers & 17 & 7 & 20 \\
\hline
\end{tabular}

The outcome measure is Hedges' $g$, a standardized effect size corrected for small sample sizes. All analyses were conducted in R [17] using the package metafor [18] implementation of multivariate random effects models.

We inspected both datasets to make sure that all data points that we would eventually analyze should be conceptually included. We excluded from Vowels points where nativeness was ambiguous (6 effect sizes), either because it was an allophonic distinction or because the contrast was instantiated in a dialect that was different to the infants' own. We further excluded from WordSeg data points corresponding to experiments where the stimuli were not in the infants' native language or accent (29) and where target words to be segmented were not content words (17). Experiments where infants were not typically developing and monolingual were generally excluded (21 in Vowels, 14 in WordSeg). Finally, effect sizes that were more than three standard deviations from the mean of the meta-analysis were also excluded ( 3 from Vowels, 1 from WordSeg).

We additionally inspected the meta-analyses for variables whose effect should be controlled for because they vary across studies and are likely to affect effect size. Indeed, in Vowels we controlled for whether studies involved a habituation phase, a fixed-length familiarization phase, conditioning, or neither, by introducing an additional moderator in our analyses to avoid spurious effects of method. Some experiments tested the same infants in multiple conditions and thus these data were not independent; we thus used meta-analytical regression models that controlled for shared covariance across repeated measures in our analyses.

\section{Results}

\subsection{Preliminary Analyses: Dataset Comparison}

Our research questions directly relate to age effects. As a first step, we investigated whether it is necessary to restrict analyses to studies where the exact same stimuli and procedure have been used with multiple age groups to avoid confounds. This restriction takes into account that studies targeting children of specific age groups might adjust task difficulty (e.g., by changing the stimuli or aspects of the procedure) or aim to tap into general abilities in younger infants and investigate more subtle capabilities in older children. This is not unlikely, especially when looking at Vowels, where infants between 0 and 16 months are included (cf. Figure 1). Such a restricted analysis has been used in a previous study of developmental changes in vowel discrimination [13]; and was reported complementary to full-scale analyses of word segmentation [14]. We assessed to what extent developmental conclusions are affected by the analysis strategy, using as a test case the theory-independent expectation that effect sizes should increase for native vowel discrimination and word segmentation, and decrease for non-native vowel discrimination. Results are shown in Table 2. Note that both the full datasets and the subsets cover the same respective age ranges.

Table 2: Age (centered per dataset, in months) effect both for the full dataset (all) and the restricted $(r$.) subsets; V=Vowels, $W=$ WordSeg. Significant p-values are marked in bold.

\begin{tabular}{lrrr}
\hline \multicolumn{1}{c}{ Dataset } & estimate (SE) & CI & $\boldsymbol{p}$-val \\
\hline V-Native-all & $0.001(0.016)$ & {$[-0.031,0.033]$} & .996 \\
V-Native-r. & $0.033(0.015)$ & {$[0.004,0.062]$} & $\mathbf{. 0 2 8}$ \\
V-NonNat.-all & $-0.011(0.017)$ & {$[-0.046,0.024]$} & .535 \\
V-NonNat.-r. & $-0.019(0.016)$ & {$[-0.050,0.013]$} & .251 \\
W-all & $0.006(0.008)$ & {$[-0.010,0.022]$} & .460 \\
W-r. & $0.030(0.013)$ & {$[0.004,0.055]$} & $\mathbf{. 0 2 5}$ \\
\hline
\end{tabular}

The results for the moderator age differ across datasets and depending on whether or not we consider a restricted subset of the data. Both in the case of native vowel discrimination and 


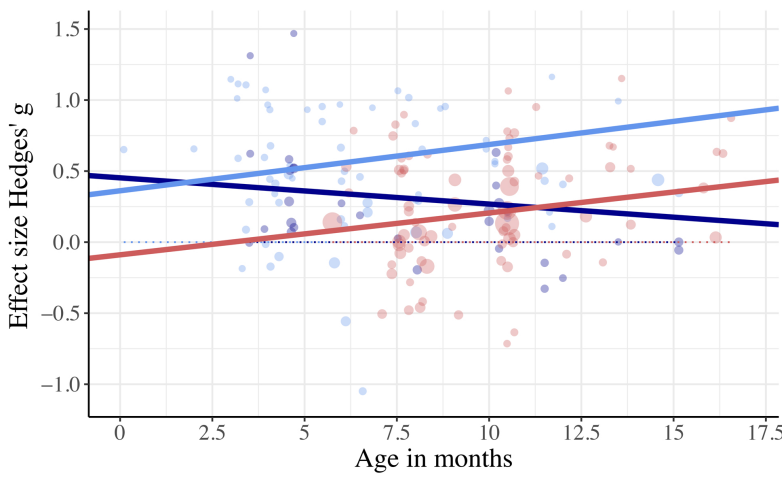

Dataset … Vowels-Native … Vowels-Nonnative … WordSeg

Figure 1: Effect size Hedges' $g$ against infant age for native vowel discrimination (dark blue), non-native vowel discrimination (light blue), and word segmentation from continuous speech (red). The dots indicate single studies, their size indicates the inverse variance by which studies are weighted in the meta-analytic regression. The lines display the meta-analytic age effect.

word segmentation we find a significant positive trend only if we use the subset of studies testing multiple age groups. Neither analysis yielded a significant effect for non-native vowels, counter to our predictions; nonetheless, the confidence interval is narrower and covers proportionally larger negative regions when using the subset compared to the whole dataset. Since these trends are the ones that were predicted in our theoryindependent validation, we conduct all subsequent analyses on the restricted datasets.

\subsection{Comparison of Age Effects across Datasets}

Figure 1 plots effect sizes against age; each point refers to an effect size stemming from a study in the respective subset of studies that tested multiple age groups in the same task. Since we used standardized effect sizes for both analyses, we can directly compare the estimates, both in the linear models and for specific ages. We observe that effect sizes are consistently higher for native vowel discrimination than for word segmentation. This pattern is consistent with the hypothesis that vowel discrimination skills emerge earlier than and independent of word segmentation skills, and also with a bottom-up interactive model where vowel categories affect word segmentation; they are not, however, consistent with top-down acquisition models.

\section{Discussion}

We set out to investigate whether the acquisition of word forms precedes infants' tuning to their native phonology - as measured by their ability to discriminate vowels - or not. The former scenario is predicted by top-down models where the ability to extract words from continuous speech a requirement for native vowel acquisition. We find no evidence for this prediction, since the experimentally attested ability to extract word forms from speech emerges at a later age than the ability to distinguish vowels, and appears to lag behind infants' tuning to their native vowel system. Our results are, however, consistent with either parallel views, where phonological and lexical knowledge develop independently, or bottom-up language acquisition theo- ries, where infants use their emerging phonological knowledge to parse the speech stream.

We discuss several (potential) limitations to these conclusions below, but before doing so we would like to point out some strengths of this study. First, our results call for further reflection and refinement of theoretical models of infant speech perception. Below, we point out specific ways in which topdown theories could be restated to accommodate the present results, but we believe these results could also spur additional work on bottom-up models of perception, which have recently fallen out of the limelight. We hope, in particular, that researchers may be able to seek direct confirmatory evidence for the precedence of phonological development compared to lexical development, for instance via longitudinal individual differences studies. Given our results, we expect that native vowel discrimination at the individual level will improve prior to, and perhaps predicts, lexical processing skills.

A second strength of the present paper lies in the novelty of the approach of re-using meta-analytic data to answer developmental questions which are at the heart of theoretical debates on infant speech perception. In previous work [16], we investigated broader patterns of development across a range of levels in language acquisition, and we expect that, as MetaLab grows, similar studies will be conducted on other aspects of development. Naturally, this extension may not be straightforward and will require careful selection of the data and reflection on potential confounds. For instance, in order to ascertain the viability of our datasets, we made sure that we could detect improvements with age predicted by all contending theories before moving on to further analyses. The data we are using have not been collected for this purpose, and since researchers have been studying a range of speech perception dimensions, it is difficult to read regression lines as purely tagging development. Notice, in particular, the divergence in results found for age when looking at all studies versus the subset of studies that contained age group comparisons.

There are (potential) limitations to our conclusions and general approach. One may criticize the coverage of the native phonological system, and particularly the use of vowel discrimination to measure phonological development. Our choice was motivated mainly by availability (a consonant meta-analysis not being yet available, not to mention other phonological units such as prosody) and further justified by the fact that vowels have been at the center of modeling studies establishing a possible proto-lexical influence on phonological acquisition $[8,9,10]$.

Nonetheless, for several reasons, assessing only vowel discrimination may be insufficient. First, vowels are thought to be special in the phonological system, such that non-native or within-category distinctions remain discriminable even for adults [19], leading to a more continuous representation than consonants. In line with this interpretation, we failed to find a significant age-related decline, even when we restricted our analyses to the subset of data where groups of infants differing in age were tested with the exact same stimuli. If anything, this aspect of vowel perception should have worked against us: If vowels are less affected by native language, then we should see smaller effects overall, rather than earlier and stronger effects.

Second, among adults vowels have been shown to be less important than consonants to identify the meaning of a word [20], which may make them a poor testing case of interactive models of language acquisition. However, recent work suggests that vowels are more important than consonants in lexical recognition for infants: When 5-month-olds are presented with 
mispronunciation of the onset of their own name, which either began with a vowel or a consonant, infants' looking patterns suggested that own-name recognition is disrupted to a greater extent by the vowel change than the consonant change [21] Thus, at least at young ages, vowels seem a reasonable choice for assessing potential phonology-lexicon interactions.

Third, using a vowel meta-analysis might be insufficient because in many languages, and certainly for the languages most infants in our databases were learning, consonants form the majority of the phonological system. We therefore are not measuring acquisition of the whole phonological system. In fact, previous research suggests a different time line of acquisition for vowels and consonants, the latter emerging later in development, and thus potentially closer to the ages at which we observe word segmentation skills emerging [3, 22]. It is unclear why language-specificity is apparent in vowels earlier than in consonants; some have proposed that since vowels are perceivable in utero, experience begins to accumulate before birth [23], whereas presumably for many consonants, and the consonantvowel sequences that constitute words, it is necessary to wait until birth for experience to accumulate. While we admit that this last argument is fair, it does not necessarily invalidate our case. As we show, vowel sensitivity, a crucial phonological skill, emerges prior to, and potentially independently from, lexical skills. To reconcile our findings with top-down theories that propose that the lexicon plays a central role in phonological acquisition, these theories might have to be reformulated more precisely, for example as "the lexicon plays a central role in consonantal acquisition".

Was our choice of word segmentation justified? It might well be that an inventory of word forms is not the type of knowledge at play during top-down phonological acquisition. Instead, infants might rely on word-meaning mappings, among other things to avoid relying on possibly wrongly segmented word forms [11]. One of our motivations was availability, as metaanalyses on word-to-meaning mapping are still under development. But more importantly, our choice was justifiable in view of current literature: As discussed in the Introduction, a protolexicon composed of word forms has been put forward as the primary source of constraints on infants' phonological knowledge by several theorists. To take a specific example, Feldman et al. [8] propose that infants may be able to use the fact that a vowel occurs in two different lexical contexts in order to separate them; and substantiates this claim with a study that shows such lexical processes being at play in a lab setting for 8-montholds already. Top-down theories could well be restated in ways compatible with our data; for instance, one may say this process relies on word forms in infants' long-term memory, and does not directly depend on the ability to extract word forms from continuous speech. Indeed, the word segmentation metaanalysis is largely based on laboratory experiments where infants are exposed to just a couple of word forms repeatedly, and are then immediately tested on passages containing those word forms and new ones (or the opposite: familiarized with passages, tested with word forms). In the whole database, only 6 studies delay the test phase and/or use exposures in the child's home; and none of them were part of the final analyses given our constraint of including only studies that measured two age groups with the same stimuli. Further, since infants are usually familiarized and tested in close succession, it might be sufficient for them to perform a sort of phonetic pattern matching, which need not employ symbolic representations and/or their long-term memory [24]. We hope future work will help address that this important gap in the current literature.
Regarding our methodological approach, there is one issue that tempers our enthusiasm for the findings, namely that one cannot be sure that tasks across domains have been matched in difficulty. Indeed, what we observe as a developmental lag could be an index of the fact that vowel discrimination tasks (at least in the ways in which they have been implemented in this literature) are intrinsically easier than word segmentation tasks (with the same caveat applying here). If tasks were truly matched and only skills varied with time, we might expect effect sizes for word segmentation to eventually be at exactly the same level as for native vowel discrimination. We cannot test this empirical question, however, because word segmentation has been tested with familiarization-based paradigms exclusively, while native vowel discrimination specifically has not been tested using familiarization for infants older than 9 months (at least in our database). Nonetheless, we do not see our approach discredited by this open empirical question, and it is not the case that one can only meta-analyze for developmental effects when the task has been held constant. In fact, this would make the study of precedence across phenomena impossible: The exact same task can never be used to test vowel discrimination and word segmentation.

Finally, we advise caution regarding potential generalizations of the present approach to other developmental and speech perception questions. As explained in the Methods and Results sections, the meta-analyst must be careful and transparent when considering study inclusions and exclusions. Additionally, meta-analyses by definition can only as good as the data they contain. It is particularly problematic if a meta-analysis builds on studies that are poor in quality, for instance, having overall low signal to noise ratios due to being underpowered. Ongoing analyses of MetaLab suggest this is likely the case in most infant literature [15]. Other aspects seem to us to be less saliently an issue; for instance, if a meta-analysis contains data selection and/or publication bias, this need not per se challenge the conclusions found. To take a specific example, the native vowel discrimination meta-analysis has significant funnel plot asymmetry [13], one of the hallmarks of publication bias, whereby researchers tend to under-report studies with effects that are small or go in a counter-intuitive direction. Nonetheless, this bias for larger effect sizes should apply across age groups and is thus entirely orthogonal to our question regarding the ages at which these skills are developed.

\section{Conclusions}

We approached a fundamental theoretical question regarding the possible top-down effects of lexical acquisition onto phonological development. Our meta-analytic results do not support such models, being more compatible instead with bottom-up or parallel theories of the development of early speech perception. We pointed out some apparent and real limitations of these conclusions, and defined promising avenues for further work.

\section{Acknowledgements}

The present work was supported by the H2020 European Research Council [Marie Skłodowska-Curie grant Nos 660911 and 659553], the Agence Nationale pour la Recherche [ANR14-CE30-0003 MechELex, ANR-10-IDEX-0001-02 PSL*, ANR-10-LABX-0087 IEC], the Fondation de France, the Berkeley Initiative for Transparency in Social Sciences and the Laura and John Arnold Foundation. 


\section{References}

[1] J. F. Werker and R. C. Tees, "Cross-language speech perception Evidence for perceptual reorganization during the first year of life," Infant Behavior and Development, vol. 7, no. 1, pp. 49-63, 1984.

[2] P. K. Kuhl, K. A. Williams, F. Lacerda, K. N. Stevens, and B. Lindblom, "Linguistic experience alters phonetic perception in infants by 6 months of age," Science, vol. 255, no. 5044, pp. 606-608, 1992.

[3] P. K. Kuhl, "Early linguistic experience and phonetic perception: Implications for theories of developmental speech perception," Journal of Phonetics, vol. 21, pp. 125-139, 1993.

[4] G. K. Vallabha, J. L. McClelland, F. Pons, J. F. Werker, and S. Amano, "Unsupervised learning of vowel categories from infant-directed speech," Proceedings of the National Academy of Sciences, vol. 104, no. 33, pp. 13273-13 278, 2007.

[5] J. F. Werker, H. H. Yeung, and K. A. Yoshida, "How do infants become experts at native-speech perception?" Current Directions in Psychological Science, vol. 21, no. 4, pp. 221-226, 2012.

[6] R. Tincoff and P. W. Jusczyk, "Some beginnings of word comprehension in 6-month-olds," Psychological Science, vol. 10, no. 2, pp. 172-175, 1999.

[7] E. Bergelson and D. Swingley, "At 6-9 months, human infants know the meanings of many common nouns," Proceedings of the National Academy of Sciences, vol. 109, no. 9, pp. 3253-3258, 2012.

[8] N. H. Feldman, E. B. Myers, K. S. White, T. L. Griffiths, and J. L. Morgan, "Word-level information influences phonetic learning in adults and infants," Cognition, vol. 127, no. 3, pp. 427-438, 2013.

[9] A. Martin, S. Peperkamp, and E. Dupoux, "Learning phonemes with a proto-lexicon," Cognitive Science, vol. 37, no. 1, pp. $103-$ 124, 2013.

[10] D. Swingley, "Contributions of infant word learning to language development," Philosophical Transactions of the Royal Society of London B: Biological Sciences, vol. 364, no. 1536, pp. 36173632, 2009.

[11] C. Ngon, A. Martin, E. Dupoux, D. Cabrol, M. Dutat, and S. Peperkamp, "(Non) words, (non) words, (non) words: Evidence for a protolexicon during the first year of life," Developmental Science, vol. 16, no. 1, pp. 24-34, 2013.

[12] S. Tsuji, C. Bergmann, and A. Cristia, "Community-augmented meta-analyses: Toward cumulative data assessment," Perspectives on Psychological Science, vol. 9, no. 6, pp. 661-665, 2014.

[13] S. Tsuji and A. Cristia, "Perceptual attunement in vowels: A metaanalysis," Developmental Psychobiology, vol. 56, no. 2, pp. 179 191, 2014.

[14] C. Bergmann and A. Cristia, "Development of infants' segmentation of words from native speech: A meta-analytic approach," Developmental Science, vol. 19, no. 6, pp. 901-917, 2016.

[15] C. Bergmann, S. Tsuji, P. E. Piccinini, M. L. Lewis, M. Braginsky, M. C. Frank, and A. Cristia, "Assessing experimental practices in language acquisition research through meta-analyses," 2017, Manuscript under review. [Online]. Available: https://osf.io/ uhv3d/?view_only=2a78e7f3157443db964ac0c69dfeee94

[16] M. L. Lewis, M. Braginsky, S. Tsuji, C. Bergmann, P. E Piccinini, A. Cristia, and M. C. Frank, "A quantitative synthesis of early language acquisition using meta-analysis," Jan 2017, preprint available on PsyArXiv. [Online]. Available: osf.io/pcqac/

[17] R Core Team, R: A Language and Environment for Statistical Computing, R Foundation for Statistical Computing, Vienna, Austria, 2016. [Online]. Available: https://www.R-project.org/

[18] W. Viechtbauer, "Conducting meta-analyses in R with the metafor package," Journal of Statistical Software, vol. 36, no. 3, pp. 1-48, 2010. [Online]. Available: http://www.jstatsoft.org/v36/i03/

[19] D. B. Fry, A. S. Abramson, P. D. Eimas, and A. M. Liberman, "The identification and discrimination of synthetic vowels," Language and Speech, vol. 5, no. 4, pp. 171-189, 1962.
[20] A. Cutler, N. Sebastián-Gallés, O. Soler-Vilageliu, and B. Van Ooijen, "Constraints of vowels and consonants on lexical selection: Cross-linguistic comparisons," Memory \& Cognition, vol. 28, no. 5, pp. 746-755, 2000.

[21] C. Bouchon, C. Floccia, T. Fux, M. Adda-Decker, and T. Nazzi, "Call me Alix, not Elix: Vowels are more important than consonants in own-name recognition at 5 months," Developmental Science, vol. 18, no. 4, pp. 587-598, 2015.

[22] P. W. Jusczyk and R. N. Aslin, "Infants' detection of the sound patterns of words in fluent speech," Cognitive Psychology, vol. 29, no. 1, pp. 1-23, 1995

[23] C. Moon, H. Lagercrantz, and P. K. Kuhl, "Language experienced in utero affects vowel perception after birth: A two-country study," Acta Paediatrica, vol. 102, no. 2, pp. 156-160, 2013.

[24] C. Bergmann, L. ten Bosch, P. Fikkert, and L. Boves, "A computational model to investigate assumptions in the headturn preference procedure," Frontiers in Psychology, vol. 4, 2013. 